\title{
Safe Lines to Life Savers-A Focus on Rescuer Safety
}

\author{
Ashima Sharma $^{1}$ Sreelekha Narla ${ }^{1}$ Shalini Pasupuleti ${ }^{1}$ \\ ${ }^{1}$ Department of Emergency Medicine, Nizam's Institute of Medical \\ Sciences, Panjagutta, Hyderabad, Telangana, India62123129

\begin{abstract}
Address for correspondence Ashima Sharma, MD, Department of Emergency Medicine, Nizam's Institute of Medical Sciences, Panjagutta, Hyderabad, Telangana 500082, India (e-mail: ashimanims@gmail.com).
\end{abstract}

Ind J Car Dis Wom 2021;6:123-129.

\begin{abstract}
Keywords

- airway management during resuscitation

- COVID-19 pandemic

- CPR

- rescuer safety

Objective To describe and discuss the new cardiopulmonary resuscitation (CPR) guidelines during the coronavirus disease 2019 (COVID-19) period.

Background The ongoing COVID-19 pandemic has affected the entire humankind despite immense efforts by the government, like mandatory personal protection, lockdown, and so forth. Numerous health care workers (HCW) lay rescuers during resuscitation are at risk of acquiring infection. This resulted in the formulation of additional guidelines during CPR, specific to COVID-19.

Method A narrative overview of new resuscitation guidelines made for protecting rescuers during COVID-19 by American Heart Association (AHA), European guidelines on resuscitation update, Research Society of Anesthesiology and Clinical Pharmacology, and Indian Resuscitation Council (IRC).

Discussion An overview of all new steps of resuscitation starting from the checking responsiveness of the patient, rescuer safety, control of aerosolization, number of team members, CPR, airway management, drugs and dosages, defibrillation, appropriateness of continuing resuscitation, and discussing the changes made in standard guidelines.
\end{abstract}

\section{Introduction}

COVID-19 SARS-CoV-2 or $\mathrm{nCoV}-19$ is a member of single-stranded ribonucleotide (RNA) viruses, and the first infection outbreak was reported from Wuhan, Hubei province, China, in November 2019. The World Health Organization (WHO) declared the coronavirus outbreak as a pandemic on March 11. 2020.1,2 It has been a year since and the panic and frustration caused by this disease in health care workers (HCW) is significant. The transmission of this virus is mainly airborne. Direct contact with a COVID-19 patient or exposure to air droplets (generated by coughing or sneezing) are the main pathways of spread of this infection. The size of virus is 0.06 to $0.14 \mu \mathrm{m}$. Infection occurs when these droplets are inhaled or deposited on mucous membranes of nose, mouth, and eyes. Respiratory droplets also form particles when they dry in airstream. This results in airborne infection, that is community spread, which is a debatable topic in itself. Transmission occurs rapidly in enclosed spaces with poor ventilation like emergency rooms, resuscitation areas in hospital, and ambulances.

To do or not to do CPR in COVID-19 patients is a question frequently raised. The default provision of CPR can be debated in following situations ${ }^{3}$ :

- The HCW is not fully donned with personal protective equipment (PPE) in in-hospital cardiac arrest (IHCA).

- CPR is not continued if the first shock is unable to restart the heart in a shockable rhythm in out-of-hospital cardiac arrest (OHCA), according to University Hospitals Birmingham National Health Service Foundation Trust.
DOI https://doi.org/ $10.1055 / \mathrm{s}-0041-1732507$
C 2020. Women in Cardiology and Related Sciences.

This is an open access article published by Thieme under the terms of the Creative Commons Attribution-NonDerivative-NonCommercial-License, permitting copying and reproduction so long as the original work is given appropriate credit. Contents may not be used for commercial purposes, or adapted, remixed, transformed or built upon. (https://creativecommons.org/licenses/by-nc-nd/4.0/).

Thieme Medical and Scientific Publishers Pvt. Ltd. A-12, 2nd Floor, Sector 2, Noida-201301 UP, India 
- Post resuscitation, the victim will need a ventilator and an intensive care unit (ICU) bed and if that is not available in pandemic surge, it is futile to attempt resuscitation.

- If the patient's condition is deteriorating significantly despite provision of critical care (e.g., in patients with progressive refractory hypoxemia or shock despite optimal support, resuscitation would not address the underlying deterioration).

The recommendations should subscribe to the code of ethics ("fairness, the duty to care, the duty to steward resources, transparency in decision-making, consistency, proportionality, and accountability") as much as possible. Available evidence does not support categorical withholding of resuscitation from patients with particular medical conditions. Factors such as age and underlying illness have been linked to outcomes after inpatient cardiopulmonary arrest, but exclusion of patients with specific disease states, including COVID-19, is not recommended on the basis of current evidence.

A very rapid transmission of virus has resulted in the pandemic overburdening the entire health care system. Worldwide affected population is 57,909,991 and deaths are 13 lakhs till date. In India the affected population is 9,050,603 and deaths are 131,000 . The most tragic part is the loss of life of over 515 HCWs while they were providing care to COVID-19 victims. ${ }^{4}$

\section{Need for Emergence of New Guidelines}

The pandemic created a situation where COVID-19 patients outnumbered the health care providers by a large ratio, so addition of new guilines was an urgent requirement. Resuscitation involves aerosol-generating procedures like chest compressions, intubation, positive pressure ventilation like bag mask ventilation (BMV), non invasive ventilation (NIV), and suctioning. This implies that rescuer safety has moved from scene safety to multiple continuous safety links during the entire episode of CPR.

The new guidelines accept that COVID-19 viral particles can remain in air for 30 to 60 minutes to a maximum period of up to 3 hours. ${ }^{5}$ The new guidelines apply to those attending an unresponsive patient in a red-zone area (high number of COVID-19 cases reported) or a suspect and confirmed COVID-19 and do not apply for those rescuers attending COVID-19-negative patients. We will initially highlight the essential equipment including protective gear to be used by the CPR provider:

- Personal protective equipment(PPE): This would include face respirator mask, and eye and body protection. A facial respirator mask with a filter against particles sized $>0.3 \mu \mathrm{m}$ is appropriate. A FFP 2/N95 mask is commonly available to CPR teams.

- Use of automated CPR machines: Devices like LUCAS (Stryker, JoLife AB.) ensure that the HCW can initiate CPR without coming in actual contact with patients. The newer guidelines have suggested training of the doctors on use of such devices. However, it is categorically mentioned not to use them without required training.
- Use of video laryngoscopes during intubation: The process of intubation during CPR or after return of spontaneous circulation (ROSC) can expose the HCW team to major aerosolization. Regular Macintosh laryngoscopes require the operator to view the glottis by bringing his face near to the mouth of the patient. This can be avoided by use of videolaryngoscopes where the eye-screen coordination can be done from a respectable distance. This equipment has moved from the difficult intubation cart to the COVID-19 cart.

- Use of supraglottic devices: Supraglottic devices are devices inserted into the pharynx to provide oxygenation and ventilation without the need for intubation. They are less invasive than intubation and require short training. They can be inserted into the patients' throats during chest compressions. It is self-explanatory that even in the most experienced hands, intubation requires stopping the compressions for some time. SGA are widely used as primary airway management devices, rescue devices, and conduit for intubation. ${ }^{6}$

\section{Steps for CPR as Applicable to Pandemic Times}

The steps for CPR as applicable to pandemic times are listed below:

1. Scene safety: Scene safety was and is the first step before attending to an unresponsive patient as per conventional approach. No scene is safe in this pandemic. It is advised that a team of doctors on rotation should remain donned with PPE in critical areas of hospital at all times. For a bystander, using facemask, eye protection, and gloves is mandatory in the COVID-19 pandemic. There is also an advisory on limitation of persons attending to an unresponsive patient.

2. Check for responsiveness: Is done by shouting "are you ok" or tapping on the shoulder. This should be done by maintaining at least a hand's distance from the patient's face. Though social distancing is a general norm all over the world, checking for the responsiveness by "tap on shoulder" remains the same. There are no new recommendations like tap on the leg or abdomen.

3. Shout for nearby help and call for emergency response system via mobile: In an out-of-hospital Cardiac arrest (OHCA) situation, if there are two rescuers, one should stay with the patient and the other should call for the emergency response team. In an in-hospital cardiac arrest (IHCA), the first responder should activate the COVID-19 code blue protocol and allow no more than three team members for single-patient CPR.

4. ONLY check for pulse and DO NOT look for breathing: Pulse check is done for not more than 10 seconds. The listen or feel for breathing has been temporarily discounted from the CPR guidelines as this has the potential for the HCW to be exposed to viral particles. High-quality CPR is started in the absence of central pulse. The contents of high-quality CPR remain the same as 100 to 
120 compressions per minute, depth of at least $5 \mathrm{~cm}$ in adults, allowing good chest recoil and minimal interruptions in compressions. However, as regards to 2 rescue breaths after every 30 compressions, a strong advisory is published. It is suggested that the first responder can choose either of the pathway based on the circumstances ${ }^{7}$ :

- They can proceed with compressions-only life support. At the same time, any cloth can cover the nose and mouth of the victim.

- Breaths can be provided if it is possible to ensure a TIGHT seal with face mask using a bag mask device with a high-efficiency particulate air (HEPA) filter interposed in between the Adult Manual Breathing Unit (AMBU) bag and mask.

- The breaths can be delivered more safely if the provider has the expertise of insertion of a SGA device early in the CPR pathway. Additional use of filters can make it safer. Using a laryngeal airway mask with a filter has shown no aerosol spread.

- There is a concept of passive oxygenation provided to the patient under a surgical mask.

This practice is applicable for IHCA with application of high-flow nasal oxygen $\left(\mathrm{O}_{2}\right)$ cannula to the victim and covering the assembly with a medical mask. It works on the assumption that every time the chest recoils during CPR, the lungs expand cyclically and suck in the $\mathrm{O}_{2}$ passively. There are no major studies to support or refute this claim.

5. Use of automated external defibrillator (AED): Unfortunately AEDs are not easily accessible to the public in India. In hospital scenarios, mostly the manual defibrillator is available for evaluation of rhythm. However, where possible, AED should be used as per the previous basic life support (BLS) protocol including application of pads while chest compressions are happening, following the AED prompts, and resuming compressions immediately after the shock is delivered. Early defibrillation for shockable rhythm and early epinephrine in nonshockable rhythm are the most important steps in CPR algorithms.

On arrival of a properly donned advanced cardiac life support provider (ACLS) team, the steps of resuscitation become more focused on rescuer safety. While one member of the ACLS team takes over the chest compressions, the team leader starts evaluating the probability of success in resuscitation versus the risk of provider exposure. This exercise is especially important for COVID-19 patients with high Charlson's comorbidity index score. The second provider from the new team is responsible for providing $\mathrm{O}_{2}$ support to the patient with minimal aerosolization. The third member performs the following actions in sequence-attach a defibrillator, check for rhythm, and prepare to intubate. The most important step is to limit aerosolization or exposure to it.

Rescue breaths are continued with a low-pressure technique with two persons (one providing a tight seal using both hands for grip). The BMV should have a HEPA filter at the expiatory valve. Scapigliati et $\mathrm{al}^{8}$ have proposed a technique to limit aerosolization. After I-gel insertion and before checking its position, an adhesive transparent drape is done from one cheek to the opposite, wrapping the external portion of I gel. A similar drape from forehead to chin is wrapped. After a good drape is prepared it is perforated at the SGA connector, connected to HEPA filter before a self-inflating bag. If complete seal is confirmed this is an easy and low-cost adjunct to protect rescuers (-Fig. 1). Locally, we have used the Freshwrap (Hindalco ${ }^{\circledR}$ ) easily available for kitchen use as it has a good stick surface.

The technique though had raised certain concerns of delay in CPR initiation due to positioning barrier, completely occlusive barrier over face would limit any SGA device (SAD) tests (i.e., drain-tube leak test) and also limit further airway maneuver like fiber optic-aided intubation through intubatable SAD. An oxygen-filled pouch is a potential hazard as it can catch fire during defibrillation. Removal of this wrap may result in potential airway dislocation and risk of aerosolization.

A recommendation made by Azalea et al is illustrated in - Fig. 2. It has an SAD with preassembled HEPA filters for rapid, easy, and safer prehospital airway management in OHCA situations. ${ }^{9}$

While high-quality CPR is ongoing, three important updates have been added. These are early use of epinephrine in nonshockable rhythms (asystole and pulseless electrical activity [PEA]), preference of lidocaine over amiodarone in shockable rhythms (pulseless VT and VF), and consideration of endotracheal intubation early in CPR.

Epinephrine in nonshockable rhythms was recommended after initiation of chest compressions after recognition of rhythm on cardiac monitor. However, now it should be given first, immediately after the rhythm recognition, with simultaneous chest compressions. Lidocaine has been suggested in COVID-9-positive cases as there is a high chance of QT prolongations due to interactions between hydroxychloroquine and azithromycin. The recommended dose is 1 to
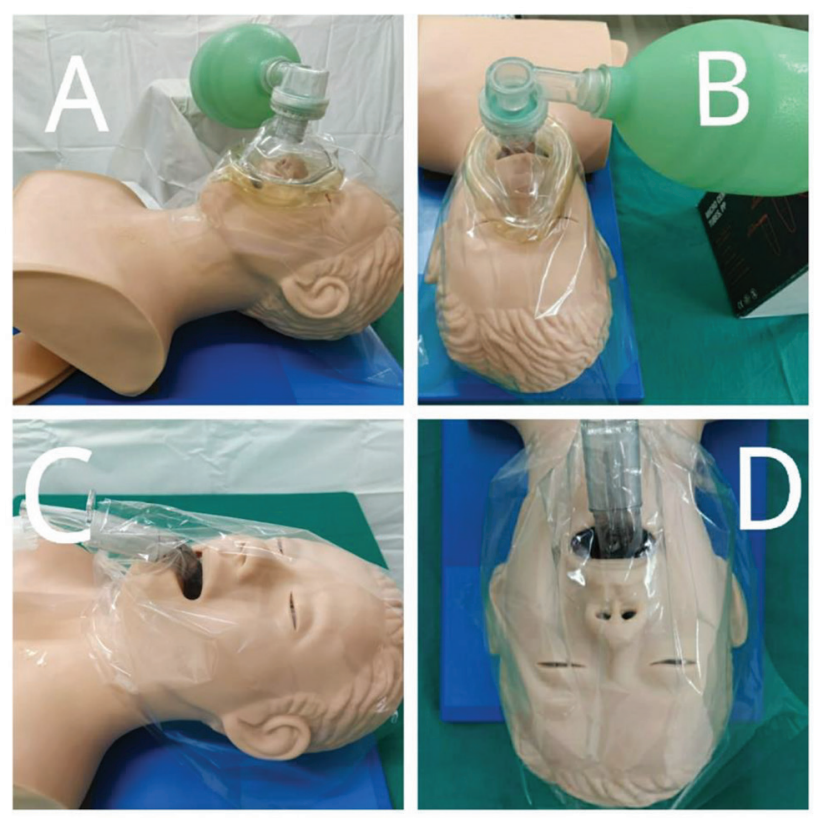

Fig. 1 Face cover of victim for limiting exposure to aerosolization. 
$1.5 \mathrm{mg} / \mathrm{kg}$ as first dose and 0.5 to $0.75 \mathrm{mg} / \mathrm{kg}$ as the second dose. As for endotracheal intubation, rapid sequence intubation is preferred. Intubation using a cuffed tube decreases the aerosol spread. Intubation is considered early for patients with COVID-19 as they cannot tolerate prolonged periods of apnea. SAS (which stands for "safe for staff and patient, accurate, swift") is the intubation drill mnemonic. If negative
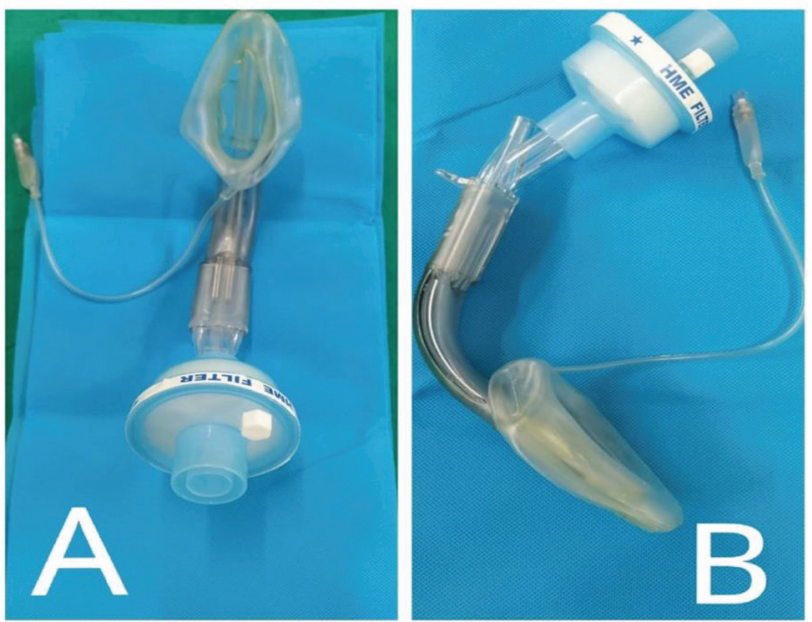

Fig. 2 Preassembled i-gel and filter for safety during rescue breaths i-gel, intersurgical. pressure rooms are available, $>12$ air changes per minute is preferred for intubation. A video laryngoscope with single-use blade should be the preferable option. After intubation, the tube should be connected to a ventilator circuit with a HEPA filter in expiratory flow. The use of closed circuits with closed inline tracheal suction catheter decreases the chances of aerosolization. Sorbello et al, however, have categorically included a step of double-checking the need for intubation in COVID-19 patients. They probably suggest the resuscitator to be sure of a good outcome before exposing themselves to the procedure. ${ }^{10}$

In our experience, use of a COVID-19 intubation box during the procedure was an additional barrier protection practiced during intubation while CPR is ongoing. The box is made of a special plastic fiber and could be slided over the head end during first pulse check (10 seconds). The two arm windows at the head end and one window each on the side allowed ventilation. To ensure that AMBU remains outside the box on the right side, we have preassembled the facemasks with a HEPA filter and a catheter mount. The AMBU bag expiratory port was connected to a corrugated tubing and an extra large (XXL) size disposal bag covered the other end of the corrugated tube. Another important issue is the limitation of the team exposed to the victim during resuscitation. In - Fig. 3, a good layout proposed by an Italian resuscitation team is projected. ${ }^{11}$

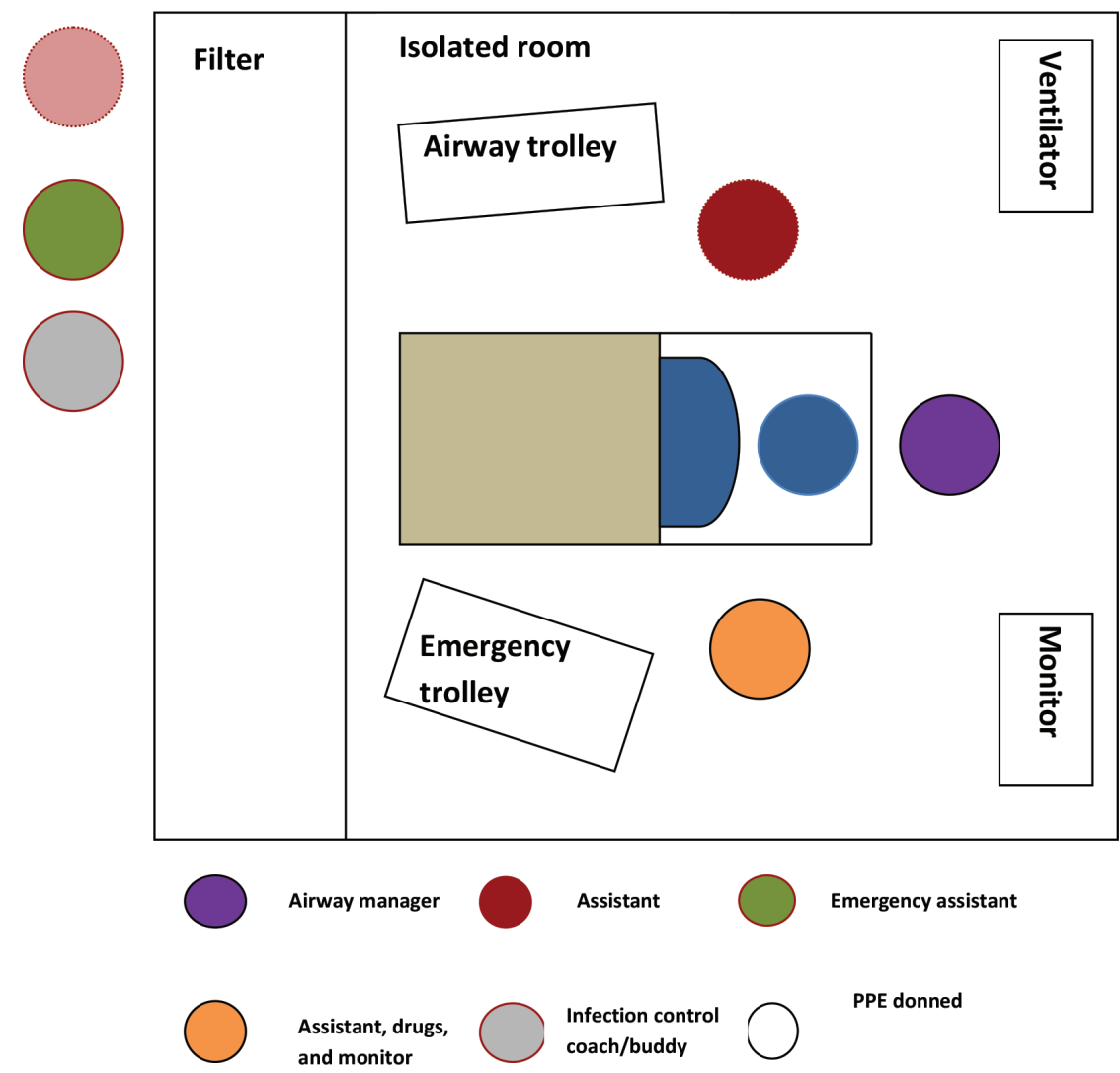

Fig. 3 Limitation of team members-a proposed layout. 


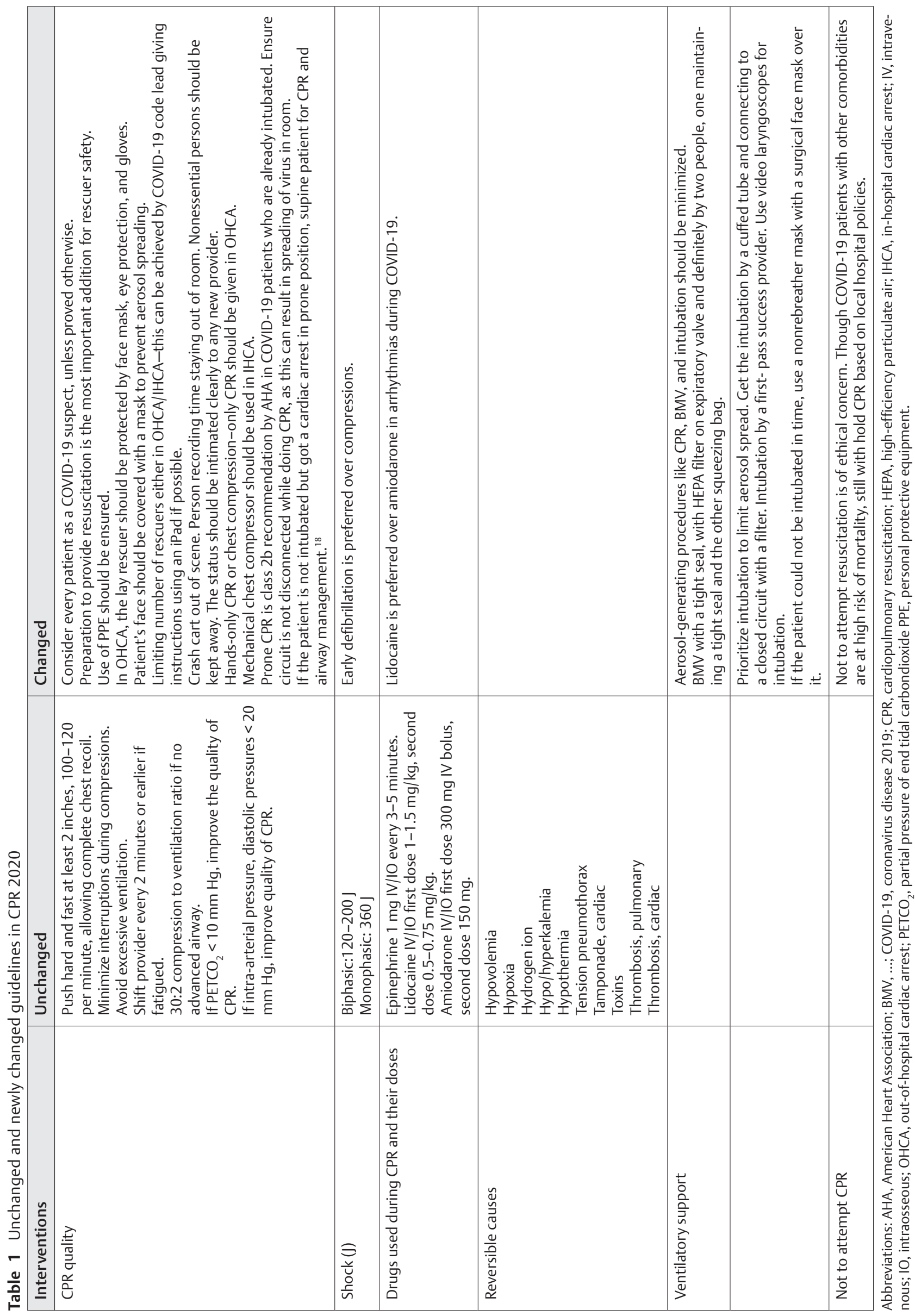


The CPR team should discuss the cause of arrest. The reversible causes of $5 \mathrm{Hs}$ and $5 \mathrm{Ts}$ remain the same as per standard CPR guidelines. The 6th and 7th $\mathbf{H}$ (hypomagnesemia and hydroxychloroquine) should be additionally considered. The post ROSC sequence focuses on the initiation of targeted temperature control and cardiorespiratory supportive interventions as before. There is a high possibility of encountering COVID-19 hypoxemia and pulmonary thrombosis, leading to cardiac arrest. Expert consultation should be sought immediately. Pressure-control ventilation to prevent barotrauma is an interesting concept immediately after connection to ventilation. PEEP (positive end expiratory pressure) for lung recruitment with low tidal volume ventilation has stood the test of time in ARDS (adult respiratory distress syndrome). It will be interesting to study the outcomes of PCV (pressured controlled ventilation) to VCV (volume controlled ventilation) in a COVID-19 lung after a successful CPR.

\section{New Interventions in CPR}

Some new interventions in CPR are discussed as follows:

- Post CPR rehabilitation: The newly added intervention of rehabilitation in CPR updates is probably most important in the COVID-19 times. A long-term plan of neurocardiopulmonary rehabilitation should be discussed with the patients' attendants and the social workers.

- The first algorithm incorporating cardiac ultrasound into CPR was introduced by Breitkreutz et al and was described as focused echocardiographic evaluation in resuscitation. ${ }^{12}$ The authors have recommended FEER after 5 cycles of high-quality CPR. We would specifically bring to the attention of our readers an article published in 2018 by Gardner et al. ${ }^{13}$ They postulated a three-step ultrasound-guided assessment of patients in cardiac arrest known as cardiac arrest sonographic assessment (CASA). Reversible causes of shock that are otherwise usually unclear during CPR can be readily identified through the use of ultrasound-such as wall motion abnormalities in patients with acute myocardial infarction. Ultrasound can also guide fluid management during ROSC by scanning the inferior vena cava and reassessing the lungs for development of pulmonary edema. ${ }^{14}$

- American Heart Association (AHA) in the 2019 update supports ECPR in patients who fail conventional CPR when the procedure can be promptly implemented and supported by skilled providers. In pandemic times, centers with lesser experience or without established ECPR programs are discouraged from initiating ECPR for OHCA. ${ }^{15}$ Experienced centers may offer ECPR for IHCA for highly selected non-COVID-19 patients depending on resource availability, while ECPR use in COVID-19positive patients requires discussion on the risk-benefit ratio. Emergency conversion of venovenous ECMO to venoarterial ECMO in the setting of a cardiac arrest in a patient receiving venovenous ECMO or during cannulation is not recommended due to the poor outcomes anticipated. The risk of infecting the HCW and contamination of clinical areas and equipment with aerosols and blood is high. The role of ECPR in resuscitation of COVID-19 patients needs to be substantiated further with research.

\section{CPR Outcomes}

The initial reports were dismal; the first study from Wuhan demonstrated just a $2.9 \%$ 30-day survival rate and a second small cohort from New York city showed absolutely no survival. Chan et al has suggested the following observationsduring the early weeks of the COVID-19 pandemic, rates of sustained return of spontaneous circulation and survival discharge decrease. ${ }^{16}$ Lower rates were seen not only in communities heavily affected by COVID-19, but also in communities with low and moderate COVID-19 mortality. The outcomes were not explained by differences in emergency medical services (EMS) arrival and treatment times, rates of bystander $\mathrm{CPR}$, or the initial rhythm, as these were adjusted for in our models. The rate of sustained return of spontaneous circulation after adjustment for demographics and cardiovascular risk factors was lower during the pandemic in 2020 compared with 2019 (23\% vs. 29.8\%; adjusted risk ratio [RR] $=0.82$; $95 \%$ confidence interval [CI], 0.78-0.87; $p<0.001$ ). Survival to discharge was $6.6 \%$ during the pandemic compared with $9.8 \%$ in 2019 ( $\mathrm{aRR}=0.83 ; 95 \% \mathrm{Cl}, 0.69-1 ; p=0.048$ ).

Hayek et al investigated IHCA in people with COVID-19 infection. The interesting findings were (i) patients who were admitted to smaller hospitals were more likely to experience cardiac arrest, (ii) admission to a smaller hospital was also independently associated with an increased risk of death among those who received CPR, and (iii) younger patients who receive CPR have reasonable survival rates compared with other critically ill patients, though less than 3\% of people older than 80 years who received CPR lived long enough to go home from the hospital. ${ }^{17}$

The unchanged and the newly changed guidelines in CPR 2020 are summarized in - Table $\mathbf{1}$.

\section{Conclusion}

This pandemic has changed risk-benefit balance for CPR: from "there is no harm in trying" to "there is little benefit to the patient and potentially significant harm to staff" 19 where the hospital staff are not provided with enhanced personal protection is justifiable. Policymakers should focus on the safety of the HCWs, especially the first responders.

\section{Conflict of Interest}

None declared. 


\section{References}

1 Centers for Disease Control and Prevention. Interim infection prevention and control recommendations for patients with suspected or confirmed coronavirus disease 2019 (COVID19) in healthcare settings. https://www.cdc.gov/coronavirus/ 2019-ncov/infection-control/control-recommendations. html. Accessed March 27, 2020

2 Silvery A, Nizami MI, Sharma A, Baskar L. Three tier screening tool and second triage to minimize the spread of COVID-19 in the emergency department of a tertiary hospital in India. Adv J Emerg Med 2020;4(2)

3 Kapoor I, Prabhakar H, Mahajan C. Cardiopulmonary resuscitation in COVID-19 patients-to do or not to? J Clin Anesth 2020;65:109879

4 Samrat S. News/DIU, India Today. Retrieved from http//www. indiatoday.liveTV.com. Accessed January 18, 2021

5 Kundra P, Vinayagam S. COVID-19 cardiopulmonary resuscitation: guidelines and modifications. J Anaesthesiol Clin Pharmacol 2020;36(Suppl 1):S39-S44

6 Malhotra N, Joshi M, Datta R, Bajwa SJ, Mehdiratta L. Indian Society of Anaesthesiologists (ISA national) advisory and position statement regarding COVID-19. Indian J Anaesth 2020;64(4):259-263

7 van Doremalen N, Bushmaker T, Morris DH, et al. Aerosol and surface stability of SARS-CoV-2 as compared with SARS-CoV-1. N Engl J Med 2020;382(16):1564-1567

8 Semeraro F, Ristagno G, Giulini G, et al. Virtual reality cardiopulmonary resuscitation (CPR): comparison with a standard CPR training mannequin. Resuscitation 2019;135: 234-235

9 Yang W-S, Hou S-W, Lee BC, et al. Taipei Azalea-supraglottic airways (SGA) preassembled with high-efficiency particulate air (HEPA) filters to simplify prehospital airway management for patients with out-of-hospital cardiac arrests (OHCA) during coronavirus disease 2019 (COVID-19) pandemic. Resuscitation 2020;151:3-5

10 Sorbello M, El-Boghdadly K, Petrini F. Airway management in COVID-19: in the den of the beast. Anesth Analg 2020;131(1):e38-e40

11 Nolan JP, Monsieurs KG, Bossaert L, et al. European Resuscitation Council COVID-Guideline Writing Groups. European Resuscitation Council COVID-19 guidelines executive summary. Resuscitation 2020;153:45-55

12 Breitkreutz R, Walcher F, Seeger FH. Focused echocardiographic evaluation in resuscitation management: concept of an advanced life support-conformed algorithm. Crit Care Med 2007;35(5 Suppl):S150-S61

13 Gardner KF, Clattenburg EJ, Wroe P, et al. The cardiac arrest sonographic assessment (CASA) exam - a standardized approach to the use of ultrasound in PEA. Am J Emerg Med 2018;36(4):729-731

14 Hussein L, Rehman MA, Sajid R, Annajjar F, Al-Janabi T. Bedside ultrasound in cardiac standstill: a clinical review. Ultrasound J 2019;11(1):35

15 Worku E, Gill D, Brodie D, Lorusso R, Combes A, Shekar K. Provision of ECPR during COVID-19: evidence, equity, and ethical dilemmas. Crit Care 2020;24(1):462

16 Chan PS, Girotra S, Tang Y, Al-Araji R, Nallamothu BK, McNally B. Outcomes for out-of-hospital cardiac arrest in the united states during the coronavirus disease 2019 pandemice-pub ahead of print. JAMA Cardiol 2020; doi10.1001/jamacardio.2020.6210

17 Hayek SS, Brenner SK, Azam TU, et al.STOP-COVID Investigators. In-hospital cardiac arrest in critically ill patients with COVID19: multicenter cohort study. BMJ 2020;371:m3513

18 Fritz Z, Perkins GD. Cardiopulmonary resuscitation after hospital admission with COVID-19. BMJ 2020;369:m1387

19 Barker J, Koeckerling D, West R. A need for prone position CPR guidance for intubated and non-intubated patients during the COVID-19 pandemic. Resuscitation 2020;151:135-136 